\title{
CRISPR/Cas9-mediated mutation revealed cytoplasmic tail is dispensable for IZUMO1 function and male fertility
}

\author{
Samantha A M Young ${ }^{1,4}$, Haruhiko Miyata', Yuhkoh Satouh¹, Masanaga Muto ${ }^{1,2}$, \\ Martin R Larsen ${ }^{3}$, R John Aitken ${ }^{4}$, Mark A Baker ${ }^{4}$ and Masahito Ikawa ${ }^{1,2}$ \\ ${ }^{1}$ Research Institute for Microbial Diseases, ${ }^{2}$ Graduate School of Pharmaceutical Sciences, Osaka University, Suita, \\ Osaka, Japan, ${ }^{3}$ Institute for Biochemistry and Molecular Biology, University of Southern Denmark, Odense, Denmark \\ and ${ }^{4}$ Priority Research Centre in Reproductive Science, Discipline of Biological Sciences, Faculty of Science and IT, \\ University of Newcastle, Callaghan, New South Wales, Australia \\ Correspondence should be addressed to M Ikawa; Email: ikawa@biken.osaka-u.ac.jp
}

\begin{abstract}
IZUMO1 is a protein found in the head of spermatozoa that has been identified as essential for sperm-egg fusion. Its binding partner in the egg has been discovered (JUNO); however, the roles of several domains within IZUMO1 remain unexplored. One such domain is the C-terminus, which undergoes major phosphorylation changes in the cytoplasmic portion of the protein during rat epididymal transit. However, the cytoplasmic tail of IZUMO1 in many species is highly variable, ranging from 55 to one amino acid. Therefore, to understand the role of the cytoplasmic tail of IZUMO1 in mouse, we utilised the gene manipulation system of CRISPR/Cas9 to generate a point mutation resulting in a premature stop codon, producing mice with truncated IZUMO1. Mice without the cytoplasmic tail of IZUMO1 showed normal fertility but decreased the amount of protein, indicating that whilst this region is important for the expression level of IZUMO1, it is dispensable for fertilisation in the mouse.

Reproduction (2016) 152 665-672
\end{abstract}

\section{Introduction}

The process of fertilisation involves a series of orchestrated, complex biological steps that culminate when the spermatozoa adhere to, and then fuse with, the oocyte plasma membrane in order to gain entry into the egg cytoplasm (Yanagimachi 1994, Gupta 2014). The processes and proteins involved in sperm-egg fusion are slowly being unraveled. Previously, our group generated an antibody against an unknown antigen, which was shown to inhibit sperm-egg fusion (Okabe et al. 1987). The antigen was identified by initial 2D-PAGE separation, and the parent protein is called 'Izumo' after the Japanese shrine dedicated to marriage (Inoue et al. 2005). Knockout studies have clearly shown that IZUMO1 is essential for male fertility, as male Izumo $1^{-/-}$ mice are infertile due to a failure of their gametes to fuse with the egg (Inoue et al. 2005).

As a testis-specific protein, IZUMO1 belongs to the immunoglobulin superfamily (IgSf) and is initially located on the outer and inner acrosomal membranes of the sperm head (Inoue et al. 2011, Satouh et al. 2012). However, during the acrosome reaction (both spontaneous and induced), IZUMO1 translocates, spreading to the postacrosomal region as well as the equatorial segment in order for fusion to take place
(Okabe et al. 1986, Okabe et al. 1987, Kawai et al. 1989, Ellerman et al. 2009, Miranda et al. 2009, Satouh et al. 2012, Sebkova et al. 2013). Although the mechanism by which this occurs is unclear, it is important to understand, as the equatorial segment is the site where sperm-egg fusion begins.

IZUMO1 contains a single transmembrane domain, and both extracellular and intracellular domains. Studies investigating the extracellular portion of IZUMO1 have clearly shown that this region binds to JUNO (formerly folate receptor 4) which is found on the surface of the plasma membrane of the egg (Bianchi et al. 2014). Female $\mathrm{Juno}^{-1-}$ mice are infertile, due to a lack of adhesion with the extracellular region of IZUMO1 on the spermatozoa. Furthermore, we have shown that the extracellular part of IZUMO1 is N-terminally glycosylated, which, whilst not essential for fertilisation, does appear to have a protective role against protein degradation during epididymal transit (Inoue et al. 2008).

Interestingly, the cytoplasmic (intracellular) side of IZUMO1 has also been suggested to play an important role in mice (Ellerman et al. 2009). The cytoplasmic region of IZUMO1 contains several potential phosphorylation sites, one of which was confirmed by Ellerman et al (2009) with the amino acid sequence SDYSGDK. Furthermore, Baker et al (2012) performed 
an in-depth proteomic analysis of the IZUMO1 peptides in rat spermatozoa and found significant post-translational changes, associated with a dynamic modification of IZUMO1 phosphorylation status during epididymal transit. All of these phosphorylation changes occurred in the cytoplasmic region of the IZUMO1 protein (Baker et al. 2012).

To investigate the role of the C-terminus of IZUMO1 in mice, we generated a mutant mouse line where the cytoplasmic portion of IZUMO1 has been removed via CRISPR/Cas9 genome editing. Our data suggest that the C-terminal, cytoplasmic region of IZUMO1 affects the expression levels of this protein yet is not essential for male fertility.

\section{Materials and methods}

\section{Animals}

All animal experiments were approved by the Animal Care and Use committee of the Research Institute for Microbial Diseases, Osaka University.

\section{Purification of phosphopeptides}

The phosphopeptides were purified using titanium dioxide $\left(\mathrm{TiO}_{2}\right)$ (Larsen et al. 2005, Jensen \& Larsen 2007) performed essentially as described in the TiSH protocol (Engholm-Keller et al. 2012) without the SIMAC step but with inclusion of a de-glycosylation step between the first and second $\mathrm{TiO}_{2}$ enrichment. The acidified phosphopeptides were purified by a reversed-phase Oligo R3 micro-column as described elsewhere (Palmisano et al. 2010) and lyophilised by vacuum centrifugation.

\section{Labelling of peptides with TMT10 plex}

Sperm peptides derived from the caput, corpus and cauda regions of the epididymis in biological duplicates were labelled with the isobaric tag TMT (Tandem Mass Tag) 10 plex reagents for simultaneous quantitation. Aliquots $(60 \mu \mathrm{g})$ of each of the peptide samples were labelled with TMT10 plex according to the manufacturer's protocol. The labelling was performed as follows: caput (TMT 127C/128N), corpus (TMT 128C/129N) and cauda (TMT 129C/130N).

\section{Hydrophilic interaction liquid chromatography (HILIC) fractionation of peptides}

The phosphopeptide sample and a reversed-phase Oligo R3 micro-column-purified non-modified peptide sample (approximately $50 \mu \mathrm{g}$ ) were re-dissolved in $40 \mu \mathrm{L} 90 \%$ acetonitrile (ACN), $0.1 \%$ trifluoroacetic acid (TFA) by first dissolving the peptides in $0.4 \mu \mathrm{L} 10 \%$ TFA, then adding $3.6 \mu \mathrm{L} \mathrm{H}_{2} \mathrm{O}$ and finally adding $36 \mu \mathrm{L} \mathrm{ACN}$. The samples were injected onto an in-house-packed TSKgel Amide-80 HILIC $320 \mu \mathrm{m} \times 170 \mathrm{~mm}$ capillary HPLC column using an Agilent 1200 capillary HPLC system. The peptides were eluted using a gradient from $90 \% \mathrm{ACN}, 0.1 \%$ TFA to $60 \% \mathrm{ACN}, 0.1 \%$ TFA for over $48 \mathrm{~min}$ at a flow rate of $6 \mu \mathrm{L} / \mathrm{min}$. The fractions were automatically collected in a microtitre plate at 1 -min intervals after UV detection at $210 \mathrm{~nm}$, and the fractions were pooled according to the UV detection to a total of 18 and 8 fractions for the phosphopeptides and non-modified peptides respectively. The fractions were dried by vacuum centrifugation. Prior to LC-MS/MS the samples were re-dissolved in $5.5 \mu \mathrm{L} 0.1 \%$ TFA. A total of $5 \mu \mathrm{L}$ of each fraction were analysed by reversedphase nanoLC-MS/MS.

\section{NanoLC-MS/MS and data analysis}

The peptides were loaded onto an Easy-nanoLC (Thermo Fisher Scientific) coupled to a Q-exactive HF mass spectrometer (Thermo Fisher Scientific). Peptides were loaded onto a precolumn $(4 \mathrm{~cm}$ Reprosil - Pur C18 AQ $5 \mu \mathrm{m}$ RP material (Dr. Maisch, Ammerbuch-Entrigen, Germany)) using the EASY-LC system and eluted directly onto a $20 \mathrm{~cm}$ long fused silica capillary column $(75 \mu \mathrm{m}$ ID) packed with Reprosil - Pur C18 AQ $3 \mu \mathrm{m} \mathrm{RP}$ material. The peptides were separated using a gradient from $1-28 \%$ B (A buffer: $0.1 \%$ formic acid (FA); B buffer: $90 \% \mathrm{ACN} / 0.1 \% \mathrm{FA}$ ) at a flow rate of $250 \mathrm{~nL} / \mathrm{min}$ over 50-110 min and then $28-45 \%$ B for $10 \mathrm{~min}$. Peptides $(\mathrm{m} / \mathrm{z}$ 450-1600) were analysed in full MS mode using a resolution of $120,000 \mathrm{FWHM}$ at $200 \mathrm{~m} / \mathrm{z}$. A maximum of 15 peptides were selected and fragmented using HCD per MS spectrum, and the fragment ions were recorded in the orbitrap using a resolution of 60,000 FWHM. Ion filling time was set to 100 $200 \mathrm{~ms}$ depending on the sample and an AGC target value of $1 \mathrm{E} 5$ ions.

\section{Data analysis}

The raw data from the Q-exactive HF were processed using the Proteome Discoverer 2.1 using the TMT10 plex quantitation method. The data were searched using both Mascot and Sequest HT search engines. For the Mascot database search the data were searched in the Sprot database with mouse as entry, with phosphorylation on S/T/Y, N-terminal acetylation as variable modifications and TMT ( $\mathrm{N}$-terminal amines and lysine) and carbamidomethyl (cysteine) as fixed modifications. For all searches the mass accuracy was set to $10 \mathrm{ppm}$ for MS data and $0.05 \mathrm{kDa}$ for MS/MS analysis.

\section{Plasmid and oligonucleotide preparation}

By injecting a sgRNA/Cas9-expressing plasmid and oligonucleotide into the pronucleus of a zygote, we generated mice with the insertion of $\mathrm{T}$ near the end of the transmembrane region, creating a stop codon. The CRISPR strategy and preparation was as follows: the plasmids expressing hCas9 and sgRNA were prepared by ligating oligos into the Bbsl site of pX330 (http://www.addgene. org/42230/; (Cong et al. 2013). The pCAG-EGxxFP reporter plasmid was prepared as described previously (Mashiko et al. 2013). A single stranded oligonucleotide containing the desired mutation with approximately $60 \mathrm{nts}$ homozygous to the target region on either side was designed and ordered. The sequence of oligonucleotide is 5'-GATCTGACTTGG 
ATCCTGACGCGGTGGGTTTGACTTCGTCACTTGCATTC TTCAATTTAGCGCTAGACTTTCCTAAAGTGAAGTACСCT GGGGCAGGACATAGGTGCACAGAACCACATTAGG

TCACAGGT-3' (insertion is lower case and italicised).

\section{Off-target analysis}

Potential off-target sites were found using free software, Bowtie (http://bowtie-bio.sourceforge.net/index.shtml) with rules outlined previously (Mali et al. 2013, Wang et al. 2013, Yang et al. 2013). Twelve to fourteen bases preceding the PAM sequence with AGG, GGG, CGG and TGG were aligned with the mouse genome (mm9). sgRNA with less number of potential off-target sites were chosen for pX330 plasmid construction.

\section{sgRNA validation assay}

Validation of sgRNA/CRISPR cleavage activity was done by transfection of HEK293T cells as reported previously (Mashiko et al. 2013).

\section{Pronuclear injection}

B6D2F1 female mice were superovulated and mated with B6D2F1 males, and fertilised eggs were collected from the oviduct. The pX330 plasmids and oligonucleotides were injected into one of the pronuclei at 5 and $100 \mathrm{ng} / \mu \mathrm{L}$ respectively. The sgRNA sequence used for injection was 5'-gTTCCTAAAGTGAAGTACCCT-3' (the initial ' $\mathrm{g}$ ' was added as at the time of generation this was thought to be necessary for sgRNA expression driven by U6 promoter). The injected eggs were cultivated in potassium simplex optimisation medium (kSOM) (Ho et al. 1995) overnight and then two-cell stage embryos were transferred into the oviducts of pseudopregnant ICR females. The pups were genotyped by PCR and subsequent sequence analysis.

\section{Genotyping}

For genotyping of $\Delta$ Cyt mutants, PCR was performed using following primers (Forward: aagaattcGGATGGATGGAAAGGAAACG; reverse: aagatatcGG AGCCAGAAGTCAGAACCA; initial sequence has an ' $a$ ' nucleotide followed by the restriction enzyme sequence for EcoRI (forward) and EcoRV (reverse), as these primers were also used in sgRNA validation). The PCR product was then digested with Bfal restriction enzyme. Mutant mice were assigned labels as follows: B6D2-Izumo1<em1(S346Stop) Osb $>$. They have been deposited into the Riken BioResource Centre along with the plasmid used to create them; pX330-Izumo1\#2.

\section{Quantitative PCR (qPCR)}

Mouse cDNA was prepared from testis. Each cDNA was subjected to quantitative RT-PCR analysis with a Thermal Cycler Dice TP800 (Takara Bio) using SYBR Premix Ex Taq II RR820S (Takara Bio). The relative expression level of each target gene mRNA was normalised to the amount of $b$-actin control. Each reaction was performed in triplicate and data are presented as the mean plus S.D. Primer sets were as follows: 5'-CTGCTCAGTTGGCATCGTGC-3' and 5'-GGAGTCTCGTGCTCCTCTTG-3' for Izumo 1, 5'-AAGTG TGACGTTGACATCCG-3' and 5'-GATCCACATCTGCTGGAA GG-3' for b-actin.

\section{Immunoblot analysis}

Immunoblot analysis was performed as described previously (Inoue et al. 2008). Samples were subjected to sodium dodecyl sulphate PAGE followed by western blotting under non-reduced conditions. For IZUMO1, antibody mAb No. 34 was used (Inoue et al. 2013). Anti-acetylated tubulin antibody (T7451, Sigma-Aldrich) was used as a loading control. Immunoreactive proteins were detected by an ECL western blotting detection kit (GE Healthcare,). The densitometric analyses were performed using the Image 1.44p software (Wayne Rasband, National Institutes of Health).

\section{Immunolocalisation of IZUMO1 and spontaneous acrosome reaction rates}

Visualisation of IZUMO1 using confocal microscopy and deconvolution analysis was done as reported previously (Satouh et al. 2012).

To assess spontaneous acrosome reaction rates the spermatozoa prepared from cauda epididymis were incubated in a drop of Toyoda, Yokoyama and Hamada (TYH) media (Toyoda et al. 1971) for $2 \mathrm{~h}$ at $37^{\circ} \mathrm{C}, 5 \% \mathrm{CO}_{2}$ then spotted onto slides, air dried, fixed with $4 \%$ paraformaldehyde for $5 \mathrm{~min}$ and washed in PBS for $5 \mathrm{~min}$. Non-specific protein binding sites were blocked with 5\% BSA/PBS with $0.1 \%$ Tween 20 at room temperature for $1 \mathrm{~h}$. The slides were then incubated at $4^{\circ} \mathrm{C}$ overnight with anti-IZUMO1 (Inoue et al. 2005) antibody or anti-CD46 antibody (KS107-116) that was established in our laboratory as a rat anti-mouse sperm antigen monoclonal antibody. Next, slides were incubated in the dark with Alexa Fluor 488 or 546-conjugated anti-rabbit or anti-rat IgGs respectively (Invitrogen) at room temperature in 5\% BSA/PBS with $0.1 \%$ Tween 20 . After washing, the slides were mounted in PBS. The stained cells were observed under a fluorescence microscope. Acrosome reaction rate was calculated by counting the percentage of cells displaying IZUMO1 localised over the full sperm head - confirmed by CD46 localisation indicating the acrosome reaction had indeed taken place.

\section{In vivo and in vitro male fertility tests}

Sexually matured Izumo1 mutant male mice were caged with 2-month-old B6D2F1 female mice for 3 months, and the number of pups was counted at the day of birth. In vitro fertilisation (IVF) analysis was done as reported previously (Inoue et al. 2005).

\section{Zona-free fusion assay}

Zona-free fusion assay was performed as reported previously (Inoue et al. 2005), with some minor changes. In brief, the 


\section{A}

SVLHFRKVSAKLKNASDEVKPTASGSKSDQSLSQQMGLKKASQADFNSDYSGDKSEATEN

B

LKNASDEVKPTASGSK
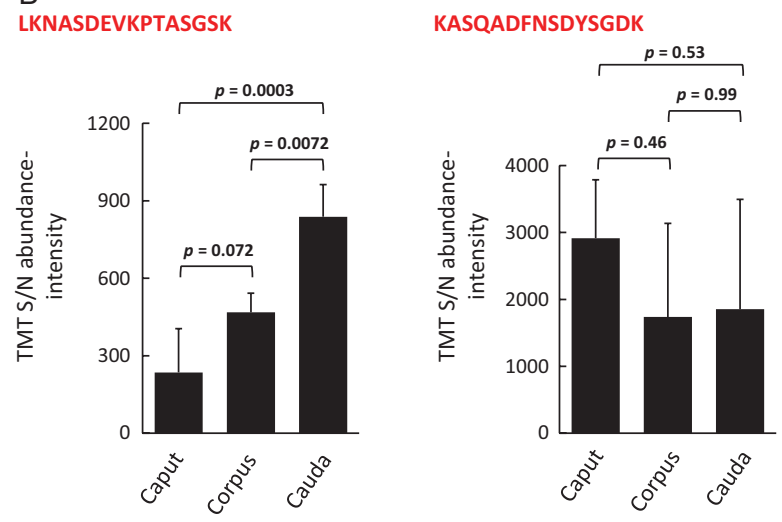

Figure 1 Phosphopeptide changes in the cytoplasmic tail of IZUMO1. (A) Amino acid sequence of the end of the transmembrane region (indicated by black line above sequence) and cytoplasmic tail of IZUMO1. The red characters indicate the peptides that were analysed in (B). (B) There were significant increases in the peptide

(LKNASDEVKPTASGSK) with 1 phosphorylation event of a serine or a threonine. In contrast, no difference or rather a decrease was observed in the peptide (KASQADFNSDYSGDK) with 1 phosphorylation event of a serine, a threonine, or a tyrosine.

zona pellucida was removed with $1 \mathrm{mg} / \mathrm{mL}$ collagenase. Zona-free oocytes were then preloaded with Hoechst 33342 by incubation in TYH media containing the dye $(1 \mu \mathrm{g} / \mathrm{mL})$ for $10 \mathrm{~min}$, then washed thoroughly. Spermatozoa were added to a concentration of $2 \times 10^{4}$ spermatozoa $/ \mathrm{mL}$. After $30 \mathrm{~min}$ of incubation the eggs were fixed with $0.25 \%$ glutaraldehyde and then observed under a fluorescence microscope (excitation with ultraviolet light).

\section{Statistical analysis}

Statistical analyses were performed using Student's $t$-test or one-way ANOVA followed by Turkey HSD test. Differences were considered significant at $P<0.05$.

\section{Results}

\section{Phosphorylation in the cytoplasmic region of mouse IZUMO1}

IZUMO1 is the essential sperm-egg fusion protein and as such, it is necessary that we understand how it is regulated. Previous studies have shown that during epididymal transit in the rat, IZUMO1 undergoes major phosphorylation changes (Baker et al. 2012). In order to determine whether phospho-changes occurred within the mouse, we enriched phosphopeptides from the spermatozoa obtained from the caput, corpus and cauda region of the epididymides. The peptides were TMT labelled and quantified using liquid chromatography coupled to mass spectrometry. Figure 1A shows a representation of the phosphopeptides observed in the
A

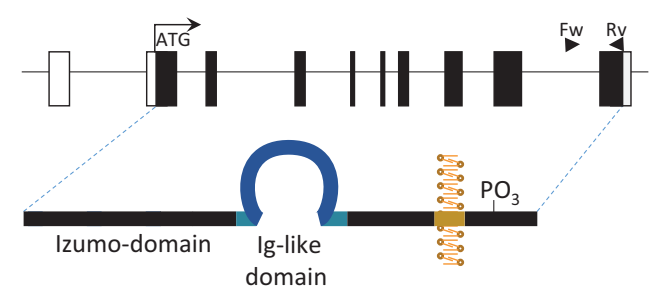

B

WT TCGGTACTTCACTTTAGGAAAGTCAGCGCTAAATTG

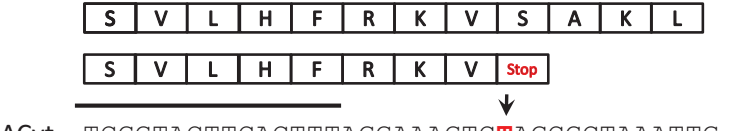

$\Delta$ Cyt

TCGGTACTTCACTTTAGGAAAGT $\underbrace{\text { CTAGCGCTAAATTG }}$
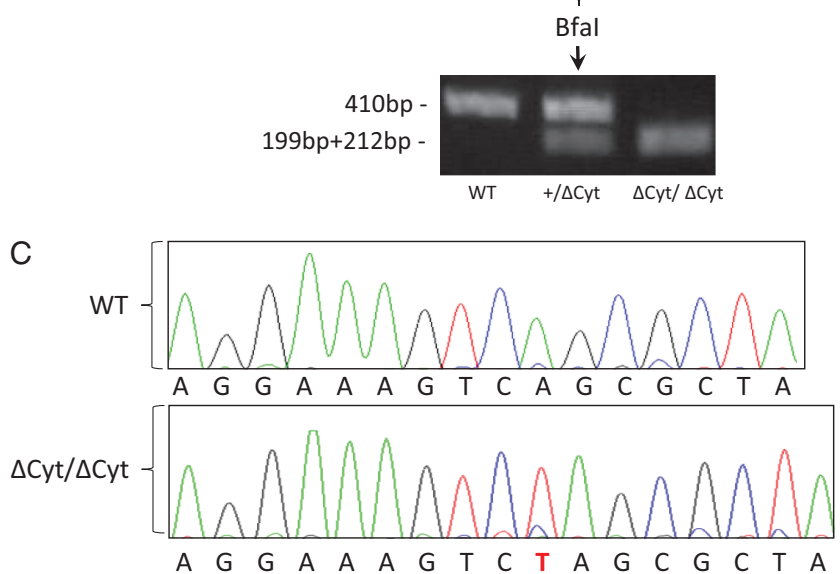

Figure 2 CRISPR strategy. (A) Structure of Izumo 1 gene and protein structure. Solid bars indicate coding exons, open bars indicate non-coding exons. Primer sequences for genotyping indicated by black arrowheads, forward (Fw) and reverse (Rv). Yellow region depicts transmembrane domain. The final coding exon of the Izumo1 gene was targeted. (B) Sequence of nucleotides and their corresponding amino acids for both WT and mutated ( $\triangle \mathrm{Cyt}$ ) Izumo1. Red lettering indicates inserted ' $\mathrm{T}$ ' nucleotide and the corresponding stop codon. Agarose gel showing Bfal digest of PCR amplicons from $W T$, heterozygote $(+/ \Delta C y t)$ and homozygote $(\Delta C y t / \Delta C y t)$ genome. Black line above sequence indicates transmembrane domain. (C) Wave pattern sequence analysis showing mutation (red ' $\mathrm{T}$ ').

cytoplasmic tail of IZUMO1. Whilst the phosphopeptide (LKNASDEVKPTASGSK) had significantly increased phosphorylations the phosphopeptide (KASQADFNSDYSGDK) remained unchanged or rather had decreased phosphorylation during epididymal transit (Fig. 1B). These data show that like the rat, IZUMO1 undergoes phosphorylation changes during epididymal maturation which may regulate this protein stability and/or function. An overview of Izumo 1 and its protein domains are shown in Fig. 2A.

\section{Generation of Izumo1 mice with truncation of the cytoplasmic tail}

In order to assess the role of the cytoplasmic domain including phosphorylation sites in IZUMO1 we removed this domain via CRISPR/Cas9 gene editing. 
This was done by the introduction of a point mutation, a single insertion of $\mathrm{T}$ nucleotide to the final exon of Izumo 1 and introducing a premature stop codon, thus producing a truncated protein (Fig. 2B and C). A total of 88 embryos were injected with 7 pups born. Of these, 4 pups were gene modified (GM). The desired mutation was inserted into 3/4 GM pups. There were no homozygous pups for the desired insertion. One mouse was heterozygous with the desired insertion and the other two carried multiple mutations as mosaics. Following selective breeding of the heterozygote mouse, the homozygous mice were obtained (Fig. 2B and $\mathrm{C}$ ). The insertion of the T nucleotide at this location also introduced a site recognised by the restriction enzyme Bfal (CTAG) (Fig. 2B). This allowed for easier genotyping for distinguishing WT and $\mathrm{KO}$ alleles.

Whilst the PCR amplicon size from WT and $\mathrm{KO}$ was $410 \mathrm{bp}$ and $411 \mathrm{bp}$, respectively, Bfal restriction enzyme digestion gave a single band of $410 \mathrm{bp}$ for WT (not digested) and single band comprised of 2 fragments (199 and 212 bp) for the KO allele (Fig. 2B).

Truncation of IZUMO1 was confirmed by immunoblot analysis, where IZUMO1 collected from the testis and from spermatozoa shifted from approximately $56 \mathrm{kDa}$ in the wild-type (WT) to approximately $40 \mathrm{kDa}$ in the homozygous samples, with the heterozygous samples showing both bands (Fig. 3A and B). An anti-acetylated tubulin antibody was used as a loading control (Fig. 3A and B). Because the amount of truncated IZUMO1 was lower than that of WT IZUMO1 in both testis and spermatozoa (Fig. 3A and $\mathrm{B}$ ), the fold change (the amount of truncated IZUMO1/WT IZUMO1) was quantified ( $n=3$ males for both WT and mutant samples). The fold change in the testis $(0.23 \pm 0.10)$ is similar to that in
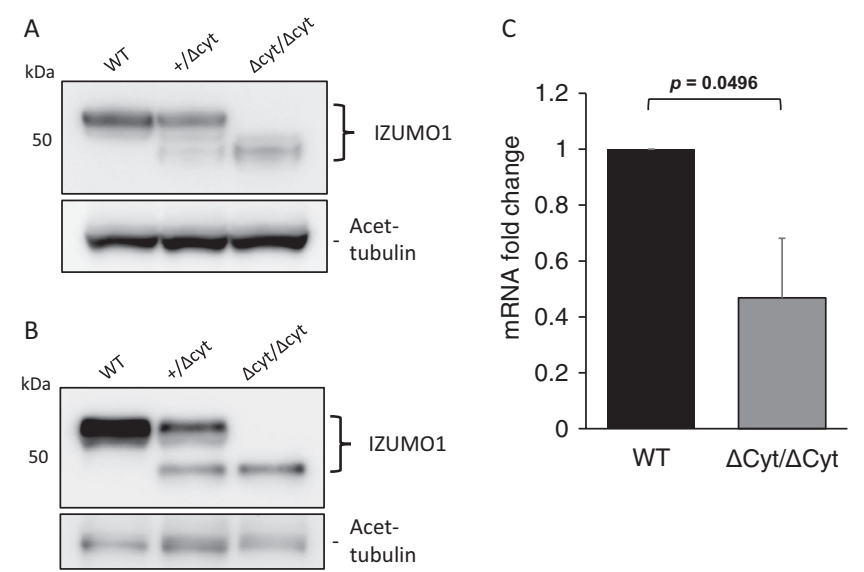

Figure 3 Truncated IZUMO1 protein expression. (A) Protein expression of IZUMO1 in WT, $+/ \Delta C y t$ and $\Delta C y t / \Delta C y t$ testis. Acetylated tubulin as loading control. (B) Protein expression of IZUMO1 in spermatozoa collected from WT, +/DCyt and $\Delta \mathrm{Cyt} / \Delta \mathrm{Cyt}$ cauda epididymis. Acetylated tubulin as loading control.

(C) Quantitative PCR analysis of WT vs $\Delta$ Cyt/ $\Delta$ Cyt Izumo1 mRNA expression. the spermatozoa collected from the cauda epididymis $(0.33 \pm 0.11)$, suggesting that the phosphorylation does not affect IZUMO1 stability during epididymal transit.

To investigate why the protein amount is already lower in the mutated testis, we performed qPCR using testis and found that the amount of Izumo 1 mRNA is lower than that of control (Fig. 3C). However, the fold change in mRNA (the amount of truncated Izumo1/WT Izumo1, $0.47 \pm 0.21$ ) was less than the fold change seen in the amount of protein (truncated IZUMO1/WT IZUMO1, $0.23 \pm 0.10$ ), suggesting that the lower protein amount of truncated IZUMO1 is caused by not only mRNA degradation but also protein instability.

A

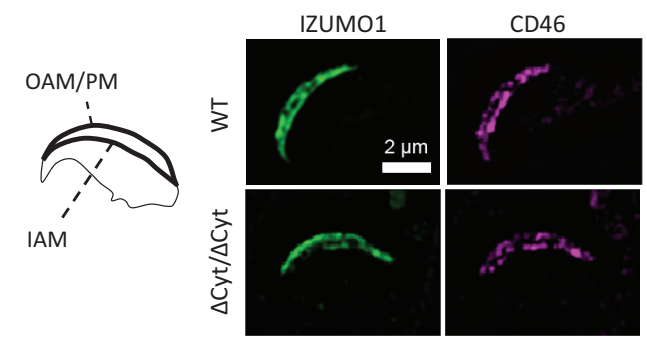

B
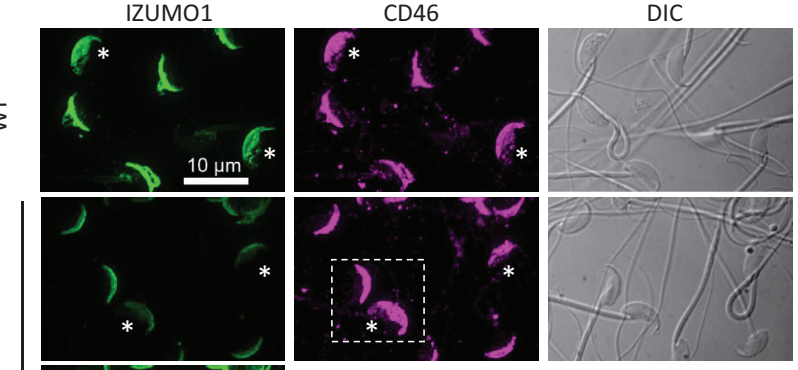

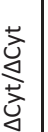
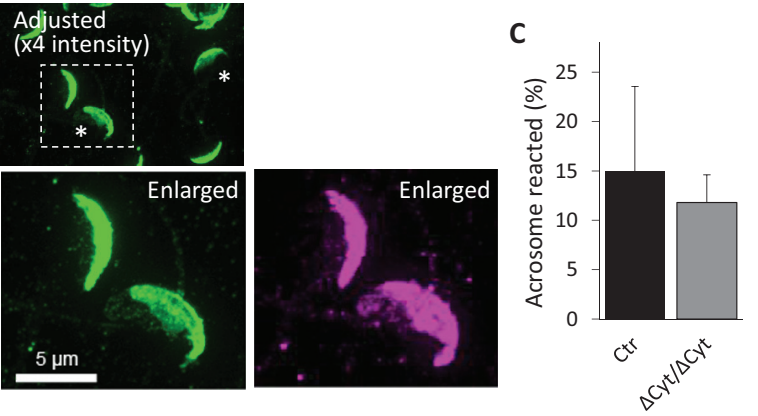

Figure 4 Immunolocalisation of IZUMO1 and spontaneous acrosome reaction. (A) Deconvolution analysis of confocal image of WT and $\Delta \mathrm{Cyt} / \Delta \mathrm{Cyt}$ IZUMO1 localisation in acrosome intact spermatozoa. CD46 as control. IAM, inner acrosomal membrane; OAM, outer acrosomal membrane; PM, plasma membrane. (B) Location of IZUMO1 over the sperm head before and after acrosome reaction in WT versus $\Delta C y t / \Delta$ Cyt spermatozoa. CD46 as a control for acrosome reaction. Asterisks indicate acrosome-reacted spermatozoa. (C) Acrosome reaction rates of control (Ctr) versus $\Delta \mathrm{Cyt} / \Delta \mathrm{Cyt}$ spermatozoa ( $n=3$ males each for Ctr and $\Delta \mathrm{Cyt} / \Delta \mathrm{Cyt})$. Percentage acrosome reacted; $15.0 \pm 8.5 \%$ vs $11.8 \pm 2.8 \%$ (Ctr vs $\Delta \mathrm{Cyt} / \Delta \mathrm{Cyt})$. 


\section{Immunolocalisation of IZUMO1 in relation to spontaneous acrosome reaction}

To analyse if the truncated IZUMO1 can translocate correctly following the spontaneous acrosome reaction (Okabe et al. 1986, 1987, Kawai et al. 1989, Sebkova et al. 2013), spermatozoa were collected and incubated for $2 \mathrm{~h}$ in TYH medium. Anti-CD46 was used as a marker of the acrosome reaction as this protein also translocates in a similar manner to IZUMO1 in human (Okabe et al. 1990, 1992) and in mouse (Fig. 4A and B). In the acrosome-intact spermatozoa, IZUMO1 is located in both the outer and inner acrosomal membranes, and translocates to the plasma membrane then spreads to cover the whole sperm head following the acrosome reaction (Satouh et al. 2012) (Fig. 4A and B). Deconvolution analysis of confocal images for IZUMO1/CD46 staining of an acrosome-intact spermatozoon showed that truncated IZUMO1 is localised in both the outer and inner acrosomal membrane (Fig. 4A). It should be noted that immunostaining of truncated IZUMO1 resulted in decreased intensity to that of WT IZUMO1, consistent with the decrease in protein amount observed with western blotting (Figs 3B, 4B and Supplementary Fig. 1 for staining control, see section on supplementary data given at the end of this article).
A
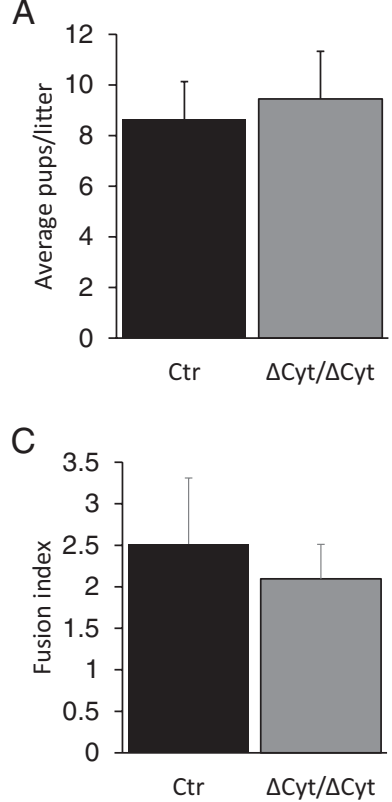

B

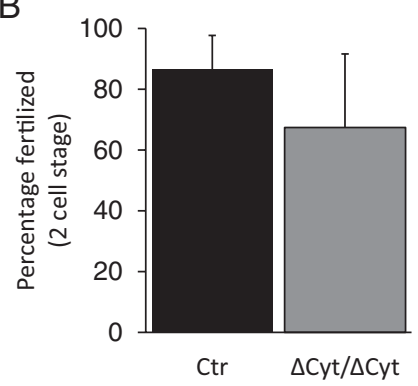

Figure 5 Analysis of fertility phenotype. (A) Litter size; $n=3$ males each for $\mathrm{Ctr}$ and $\Delta \mathrm{Cyt} / \Delta \mathrm{Cyt}$, with $2 \mathrm{WT}$ females per male. Average litter size; $\mathrm{Ctr}=8.6 \pm 1.5, \Delta \mathrm{Cyt} / \Delta \mathrm{Cyt}=9.4 \pm 1.9$ pups/litter. (B) IVF success rate; $n=3$ males each for Ctr and $\Delta$ Cyt/ $\Delta$ Cyt, Oocytes; 150 vs 195 (Ctr vs $\Delta$ Cyt/ $\Delta$ Cyt), $86.5 \pm 24.2 \%$ vs $67.4 \pm 11.2 \%$ fertilised (Ctr vs $\Delta \mathrm{Cyt} / \Delta \mathrm{Cyt}$ ). (C) Zona-free fusion success rate. Fusion index; $2.5 \pm 0.8$ vs $2.1 \pm 0.4$ (Ctr vs $\Delta \mathrm{Cyt} / \Delta \mathrm{Cyt}) ; n=3$ males each for $\mathrm{Ctr}$ and $\Delta$ Cyt/ $\Delta$ Cyt; Oocytes $=47$ vs 42 (Ctr vs $\Delta$ Cyt/ $\Delta$ Cyt); Fusion index; fused spermatozoa/egg.
However, truncated IZUMO1 showed identical localisation with WT IZUMO1 even after acrosome reaction, and co-translocation of truncated IZUMO1 and CD46 was examined further by the comparison of enhanced images of truncated IZUMO1 and CD46 (Fig. 4B). Moreover, the spontaneous acrosome reaction rate of the truncated IZUMO1 spermatozoa was comparable to that of the control mice; 11.8 and $15.0 \%$ respectively (Fig. 4C). These results indicate that the cytoplasmic region of IZUMO1 is not necessary for spontaneous acrosome reaction and subsequent IZUMO1 translocation in vitro.

\section{Male fertility of truncated Izumo1 mice}

Litter size analysis was used to determine the fertilizing ability of Izumo1 mutated mice. Mutated mice and controls were given mature WT females and allowed to copulate and breed for 3 pregnancy cycles (approx. 3 months). Pups were counted on the day of their birth. Homozygous mutated males showed comparable

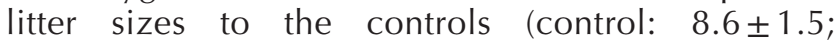
mutant: $9.4 \pm 1.9$ ) (Fig. 5A). There was no significant difference between control and mutant male mice; therefore, it was concluded that the truncated Izumo 1 mice are fully fertile. Further analysis using IVF also showed no significant difference in sperm fertilizing ability (Fig. 5B). In addition, there was no significant difference in the capacity of the spermatozoa to fuse with the plasma membrane of the oocyte in the zonafree fusion assay (Fig. 5C).

\section{Discussion}

IZUMO1 undergoes specific phosphorylation events in the cytoplasmic tail region suggesting that this region of IZUMO1 may be important for the function and/or translocation of IZUMO1 seen following the acrosome reaction. Across several mammalian species IZUMO1 exists with a highly conserved $\mathrm{N}$-terminal region, however the C-terminus differs in three ways. The cytoplasmic region can be long, as seen in rodent species such as the mouse (approx. 55 aa), shorter in humans (approx. 40 aa) and very short in porcine species - only one amino acid in length (an alanine) (Kim et al. 2013). In order to investigate the importance of this region, this study produced a mutated mouse line with a truncated version of IZUMO1. The cytoplasmic tail of the mutated mice in this study is 3 amino acids long very similar to the length seen in the pig, yet their fertility was unaffected. These data indicate that the cytoplasmic region including phosphorylation sites does not play any essential role in fertilisation in mice.

Ellerman et al. (2009) demonstrated IZUMO1's involvement in the formation of larger protein complexes. These complexes appear to be homo-tetramers, trimers 
and dimers and are formed in conjunction with different regions of the IZUMO1 protein. The extracellular domain of the protein was found to be involved in forming complexes the size of dimers, whereas the cytoplasmic tail region and/or the transmembrane region were found to form larger complexes - the trimers and tetramers (Ellerman et al. 2009). Recently, it was found that IZUMO1 is largely monomeric in spermatozoa, a structure that is altered by its binding partner, JUNO, resulting in dimerisation and tight binding between the gametes (Inoue et al. 2015). Although the cytoplasmic region may play a role in the formation of larger complexes, this region is apparently dispensable for IZUMO1 relocalisation during acrosome reaction and subsequent fusion ability.

In the pig IZUMO1 still forms complexes; however, these are mainly homodimers of IZUMO1 (Kim etal. 2013). As seen in the work by Ellerman et al (2009) complexes of this size are most likely formed by interactions between the extracellular portion of the protein, therefore it is unsurprising that pig IZUMO1 forms complexes of this size, even without a long cytoplasmic region. While the latter is clearly not necessary for the assembly of IZUMO1 complexes essential for fertility, this study has demonstrated that the C-terminus of this molecule is important in maintaining the amount of protein, however, it is not regulated by the phosphorylation changes that occur during epididymal transit.

There are many possible explanations for this impact on protein amount. One reason may be that the mRNA was unstable, leading to a decrease in levels of protein translation. Instability of the mRNA can be caused by nonsense mediated decay (NMD). However, NMD in mammals typically occurs when a premature stop codon appears more than approximately $50 \mathrm{nt}$ upstream of the junction between the final intron and exon (Hentze \& Kulozik 1999). In this case, the mutation of the Izumo 1 mRNA was in the final exon, suggesting that NMD did not play a causative role in driving down the amount of the corresponding protein in the mature gamete. That protein instability may also be involved in mediating the decreased manifestation of IZUMO1 in mutant mice is supported by comparing the relative levels of IZUMO1 expression as mRNA and protein, as set out in Fig. 3.

The discovery of the IZUMO1 binding partner JUNO, as well as the dimerisation of IZUMO1 following its recognition by JUNO, explains how the gametes are brought into close proximity during the early stages of fertilisation. Our data demonstrate that IZUMO1's cytoplasmic domain plays some roles in the regulation of IZUMO1 amount but is not involved in IZUMO1 translocation and the subsequent sperm-egg interaction.

\section{Supplementary data}

This is linked to the online version of the paper at http://dx.doi. org/10.1530/REP-16-0150.

\section{Declaration of interest}

The authors declare that there is no conflict of interest that could be perceived as prejudicing the impartiality of the research reported.

\section{Funding}

This project was supported by the JSPS KAKENHI JP25250014 (M I), JP26830056 (H M), JP24770210 (Y S), MEXT KAKENHI JP25112007 (M I), JP15K21737 (M I), JP25113714 (Y S) and Takeda Science Foundation (MI).

\section{Acknowledgements}

We thank the Department of Experimental Genome Research, Institute of Microbial Diseases, Osaka University for the generation of the mutant mice.

\section{References}

Baker MA, Hetherington L, Weinberg A, Naumovski N, Velkov T, Pelzing M, Dolman S, Condina MR \& Aitken RJ 2012 Analysis of phosphopeptide changes as spermatozoa acquire functional competence in the epididymis demonstrates changes in the post-translational modification of izumo1. Journal of Proteome Research 11 5252-5264. (doi:10.1021/ pr300468m)

Bianchi E, Doe B, Goulding D \& Wright GJ 2014 Juno is the egg Izumo receptor and is essential for mammalian fertilization. Nature $\mathbf{5 0 8}$ 483-487. (doi:10.1038/nature13203)

Cong L, Ran FA, Cox D, Lin S, Barretto R, Habib N, Hsu PD, Wu X, Jiang W, Marraffini LA et al. 2013 Multiplex genome engineering using CRISPR/ Cas systems. Science 339 819-823. (doi:10.1126/science.1231143)

Ellerman DA, Pei J, Gupta S, Snell WJ, Myles D \& Primakoff P 2009 Izumo is part of a multiprotein family whose members form large complexes on mammalian sperm. Molecular Reproduction and Development 76 1188-1199. (doi:10.1002/mrd.21092)

Engholm-Keller K, Birck P, Storling J, Pociot F, Mandrup-Poulsen T \& Larsen MR $2012 \mathrm{TiSH}$ - a robust and sensitive global phosphoproteomics strategy employing a combination of TiO2, SIMAC, and HILIC. Journal of Proteomics 75 5749-5761. (doi:10.1016/j.jprot.2012.08.007)

Gupta SK 2014 Unraveling the intricacies of mammalian fertilization. Asian Journal of Andrology 16 801-802. (doi:10.4103/1008-682X.133325)

Hentze MW \& Kulozik AE 1999 A perfect message: RNA surveillance and nonsense-mediated decay. Cell 96 307-310. (doi:10.1016/S00928674(00)80542-5)

Ho Y, Wigglesworth K, Eppig JJ \& Schultz RM 1995 Preimplantation development of mouse embryos in KSOM: augmentation by amino acids and analysis of gene expression. Molecular Reproduction and Development 41 232-238. (doi:10.1002/mrd.1080410214)

Inoue N, Hagihara Y, Wright D, Suzuki T \& Wada I 2015 Oocyte-triggered dimerization of sperm IZUMO1 promotes sperm-egg fusion in mice. Nature Communications 6 8858. (doi:10.1038/ncomms9858)

Inoue N, Hamada D, Kamikubo H, Hirata K, Kataoka M, Yamamoto M, Ikawa M, Okabe M \& Hagihara Y 2013 Molecular dissection of IZUMO1, a sperm protein essential for sperm-egg fusion. Development 140 232-238. (doi:10.1242/dev.094854)

Inoue N, Ikawa M, Isotani A \& Okabe M 2005 The immunoglobulin superfamily protein Izumo is required for sperm to fuse with eggs. Nature 434 234-238. (doi:10.1038/nature03362)

Inoue N, Ikawa M \& Okabe M 2008 Putative sperm fusion protein IZUMO and the role of N-glycosylation. Biochemical and Biophysical Research Communications 377 910-914. (doi:10.1016/j.bbrc.2008.10.073)

Inoue N, Satouh Y, Ikawa M, Okabe M \& Yanagimachi R 2011 Acrosomereacted mouse spermatozoa recovered from the perivitelline space can fertilize other eggs. PNAS 108 20008-20011. (doi:10.1073/ pnas.1116965108) 
Jensen SS \& Larsen MR 2007 Evaluation of the impact of some experimental procedures on different phosphopeptide enrichment techniques. Rapid Communications in Mass Spectrometry 21 3635-3645. (doi:10.1002/ rcm.3254)

Kawai Y, Hama T, Mayumi T, Okabe M, Matzno S, Kohama Y \& Mimura T 1989 Flow cytometric analysis of mouse sperm using monoclonal anti-sperm antibody OBF13. Journal of Reproductive Immunology $\mathbf{1 6}$ 71-82. (doi:10.1016/0165-0378(89)90007-7)

Kim E, Kim JS, Lee Y, Song BS, Sim BW, Kim SU, Saitoh T, Yazawa H, Nunoya T \& Chang KT 2013 Molecular cloning, characterization of porcine IZUMO1, an IgSF family member. Reproduction in Domestic Animals 48 90-97. (doi:10.1111/j.1439-0531.2012.02037.x)

Larsen MR, Jensen SS, Jakobsen LA \& Heegaard NH 2007 Exploring the sialiome using titanium dioxide chromatography and mass spectrometry. Molecular and Cellular Proteomics 6 1778-1787. (doi:10.1074/mcp. M700086-MCP200)

Larsen MR, Thingholm TE, Jensen ON, Roepstorff P \& Jorgensen TJ 2005 Highly selective enrichment of phosphorylated peptides from peptide mixtures using titanium dioxide microcolumns. Molecular and Cellular Proteomics 4 873-886. (doi:10.1074/mcp.T500007-MCP200)

Mali P, Yang L, Esvelt KM, Aach J, Guell M, DiCarlo JE, Norville JE \& Church GM 2013 RNA-guided human genome engineering via Cas9. Science 339 823-826. (doi:10.1126/science.1232033)

Mashiko D, Fujihara Y, Satouh Y, Miyata H, Isotani A \& Ikawa M 2013 Generation of mutant mice by pronuclear injection of circular plasmid expressing Cas9 and single guided RNA. Scientific Reports 33355. (doi:10.1038/srep03355)

Miranda PV, Allaire A, Sosnik J \& Visconti PE 2009 Localization of low-density detergent-resistant membrane proteins in intact and acrosome-reacted mouse sperm. Biology of Reproduction 80 897-904. (doi:10.1095/biolreprod.108.075242)

Palmisano G, Lendal SE, Engholm-Keller K, Leth-Larsen R, Parker BL \& Larsen MR 2010 Selective enrichment of sialic acid-containing glycopeptides using titanium dioxide chromatography with analysis by HILIC and mass spectrometry. Nature Protocols 5 1974-1982. (doi:10.1038/nprot.2010.167)

Okabe M, Adachi T, Takada K, Oda H, Yagasaki M, Kohama Y \& Mimura T 1987 Capacitation-related changes in antigen distribution on mouse sperm heads and its relation to fertilization rate in vitro. Journal of Reproductive Immunology 11 91-100. (doi:10.1016/01650378(87)90014-3)
Okabe M, Nagira M, Kawai Y, Matzno S, Mimura T \& Mayumi T 1990 A human sperm antigen possibly involved in binding and/or fusion with zona-free hamster eggs. Fertility and Sterility 54 1121-1126. (doi:10.1016/S0015-0282(16)54015-1)

Okabe M, Takada K, Adachi T, Kohama Y \& Mimura T 1986 Inconsistent reactivity of an anti-sperm monoclonal antibody and its relationship to sperm capacitation. Journal of Reproductive Immunology 9 67-70 (doi:10.1016/0165-0378(86)90026-4)

Okabe M, Ying X, Nagira M, Ikawa M, Kohama Y, Mimura T \& Tanaka K 1992 Homology of an acrosome-reacted sperm-specific antigen to CD46. Journal of Pharmacobio-Dynamics 15 455-459. (doi:10.1248/ bpb1978.15.455)

Satouh Y, Inoue N, Ikawa M \& Okabe M 2012 Visualization of the moment of mouse sperm-egg fusion and dynamic localization of IZUMO1. Journal of Cell Science 125 4985-4990. (doi:10.1242/jcs.100867)

Sebkova N, Ded L, Vesela K \& Dvorkakova-Hortova K 2013 Progress of sperm IZUMO1 relocation during spontaneous acrosome reaction. Reproduction 147 231-240. (doi:10.1530/REP-13-0193)

Toyoda Y, Yokoyama M \& Hoshi T 1971 Studies on the fertilization of mouse eggs in vitro. Japanese Journal of Animal Reproduction 16 147-151. (doi:10.1262/jrd1955.16.152)

Wang $H$, Yang $H$, Shivalila CS, Dawlaty MM, Cheng AW, Zhang F \& Jaenisch R 2013 One-step generation of mice carrying mutations in multiple genes by CRISPR/Cas-mediated genome engineering. Cell 153 910-918. (doi:10.1016/j.cell.2013.04.025)

Yanagimachi R 1994 Fertility of mammalian spermatozoa: its development and relativity. Zygote 2 371-372. (doi:10.1017/ S0967199400002240)

Yang H, Wang H, Shivalila C, Cheng A, Shi L \& Jaenisch R 2013 One-step generation of mice carrying reporter and conditional alleles by CRISPR/Cas-mediated genome engineering. Cell 154 1370-1379. (doi:10.1016/j.cell.2013.08.022)

Received 21 March 2016

First decision 21 April 2016

Revised manuscript received 5 September 2016

Accepted 13 September 2015 